\title{
NONDEGENERATE MOTION OF SINGULAR POINTS IN OBSTACLE PROBLEMS WITH VARYING DATA
}

\author{
NILES ARMSTRONG AND IVAN BLANK
}

\begin{abstract}
Recent work by Serfaty and Serra give a formula for the velocity of the free boundary of the obstacle problem at regular points ([17), and much older work by King, Lacey, and Vázquez gives an example of a singular free boundary point (in the Hele-Shaw flow) that remains stationary for a positive amount of time (13]). The authors show how singular free boundaries in the obstacle problem in some settings move immediately in response to varying data. Three applications of this result are given, and in particular, the authors show a uniqueness result: For sufficiently smooth elliptic divergence form operators on domains in $\mathbb{R}^{n}$ and for the Laplace-Beltrami operator on a smooth manifold, the boundaries of distinct mean value sets (of the type found in [7] and [5]) which are centered at the same point do not intersect.
\end{abstract}

\section{INTRODUCTION}

In his Fermi Lectures for the obstacle problem, Caffarelli stated the following theorem:

Theorem 1.1 (Mean Value Theorem for Divergence Form Elliptic PDE). Let $L$ be any divergence form elliptic operator of the form $\partial_{i}\left(a^{i j}(x) \partial_{j}\right)$ with ellipticity $[\lambda, \Lambda]$. For any $x_{0} \in \Omega$, there exists an increasing family $D_{r}\left(x_{0}\right)$ which satisfies the following:

(1) $B_{c r}\left(x_{0}\right) \subset D_{r}\left(x_{0}\right) \subset B_{C r}\left(x_{0}\right)$, with $c, C$ depending only on $n, \lambda$, and $\Lambda$.

(2) For any $v$ satisfying $L v \geq 0$ and $r<s$, we have

$$
v\left(x_{0}\right) \leq \frac{1}{\left|D_{r}\left(x_{0}\right)\right|} \int_{D_{r}\left(x_{0}\right)} v(x) d x \leq \frac{1}{\left|D_{s}\left(x_{0}\right)\right|} \int_{D_{s}\left(x_{0}\right)} v(x) d x .
$$

Finally, for any $R>0$ the set $D_{R}\left(x_{0}\right)$ is the noncontact set of the following obstacle problem:

$u \leq G\left(\cdot, x_{0}\right)$ (with $G\left(\cdot, x_{0}\right)$ denoting the Green's function) such that

$$
\begin{aligned}
& L(u)=-\chi_{\{u<G\}} R^{-n} \quad \text { in } B_{M}\left(x_{0}\right) \\
& u=G\left(\cdot, x_{0}\right) \quad \text { on } \partial B_{M}\left(x_{0}\right)
\end{aligned}
$$

where $B_{M}\left(x_{0}\right) \subset \mathbb{R}^{n}$ and $M>0$ is sufficiently large.

This theorem is a clear analogue of the classical mean value theorem for balls for the Laplacian, but here the role of the balls is replaced with the sets, $D_{r}\left(x_{0}\right)=\left\{u_{r}(x)<\right.$ 
$\left.G\left(x, x_{0}\right)\right\}$ where $u_{r}$ is the solution to Equation 1.2 with $R=r$. Of course, this theorem immediately leads to questions about exactly what can be said about these $D_{r}\left(x_{0}\right)$ 's. In fact, it is rather trivial to prove the converse of this theorem:

Theorem 1.2 (Converse MVT for Divergence Form Elliptic PDE). If $\left\{D_{r}\left(x_{0}\right)\right\}_{\left\{r>0, x_{0} \in \mathbb{R}^{n}\right\}}$ is a complete collection of sets obtained for a specific operator, L, of the form given in Theorem 1.1, and $v$ is a function such that

$$
v\left(x_{0}\right) \equiv \frac{1}{\left|D_{r}\left(x_{0}\right)\right|} \int_{D_{r}\left(x_{0}\right)} v(x) d x
$$

whenever $D_{r}\left(x_{0}\right) \subset \Omega$, then $L v=0$ in $\Omega$.

The upshot is that all of the information contained in the operator must be contained within the collection of mean value sets and vice versa.

Theorem 1.1 was proven by Blank and Hao in detail in [7], and an analogous result was shown in [5] for the Laplace-Beltrami operator on Riemannian manifolds. Since then, the authors have been studying properties of these mean value sets. Indeed, the first author showed that even for some rather simple operators, $L$, that can be chosen to be arbitrarily close to the Laplacian these sets are not necessarily convex (see [2]), and besides generalizing the result to the Laplace-Beltrami operator, the second author has shown some regularity properties of these sets (see [5] and [3]). One important question that has remained is the following one: If $y \in \partial D_{r}\left(x_{0}\right)$, then is it possible for there to exist an $\tilde{r} \neq r$ such that $y \in \partial D_{\tilde{r}}\left(x_{0}\right)$ ? One can restate this question as whether $r<s$ merely implies $D_{r}\left(x_{0}\right) \subset D_{s}\left(x_{0}\right)$, or does it imply the stronger result: $\overline{D_{r}\left(x_{0}\right)} \subset D_{s}\left(x_{0}\right)$ ? In this paper we show that in the case of the Laplace-Beltrami operator on Riemannian manifolds, and in the case where the operator $L$ has coefficients in $C^{1,1}$, the answer is yes. (When the coefficients are not $C^{1,1}$ the question is still open.)

In fact, there is almost a proof based on the Hopf Lemma that has already been pointed out in [5]. Along these lines, if we assume that $y_{0} \in \partial D_{r}\left(x_{0}\right) \cap \partial D_{s}\left(x_{0}\right)$, and we assume that $\partial D_{r}\left(x_{0}\right)$ is regular at $y_{0}$, then by invoking Caffarelli's famous free boundary regularity theorem for the obstacle problem (see [9] and/or [10]), then we are guaranteed that there will exist a ball $B_{\rho}\left(z_{0}\right)$ satisfying:

(1) $B_{\rho}\left(z_{0}\right) \subset D_{r}\left(x_{0}\right)$, and

(2) $\partial B_{\rho}\left(z_{0}\right) \cap \partial D_{r}\left(x_{0}\right) \cap \partial D_{s}\left(x_{0}\right)=y_{0}$. 
Now if we let $u_{r}$ and $u_{s}$ be the solutions to the problem in Equation 1.2 when $R=r$ and $R=s$ respectively, then it follows that $v(x):=u_{r}(x)-u_{s}(x)$ will satisfy the following:

(1) $v \geq 0$ in $B_{\rho}\left(z_{0}\right)$,

(2) $L v=s^{-n}-r^{-n}<0$ in $B_{\rho}\left(z_{0}\right)$, and

(3) $v\left(y_{0}\right)=0$.

In this situation we can apply the Hopf Lemma to guarantee that $\nabla v\left(y_{0}\right) \neq 0$. On the other hand

$$
\nabla v\left(y_{0}\right)=\nabla u_{r}\left(y_{0}\right)-\nabla u_{s}\left(y_{0}\right)=\nabla G\left(x_{0}, y_{0}\right)-\nabla G\left(x_{0}, y_{0}\right)=0
$$

which gives us a contradiction.

Of course the bad news in the "proof" above is that we assumed that $y_{0}$ was a regular point of the free boundary. Now in the most typical pictures of free boundaries with singular points, it should be even easier to touch the boundary of $D_{r}\left(x_{0}\right)$ with a ball, in spite of these examples, Schaeffer gave other examples of contact sets in the obstacle problem with cantor-like structures (see [16]) and the recent work of Figalli and Serra that yields some nice regularity results for the singular set seems to require that the operator be the Laplacian (see [1] ).

One can also ask if it is possible to repair the proof above so that it continues to hold even at the singular points, and indeed, that was our first attempt at solving this problem. In joint work by Alvarado, Brigham, Maz'ya, Mitrea, and Ziadé, a sharp form of Hopf's Lemma is shown which does not require touching with a ball; one only needs to touch with a "pseudoball" (see [1, Theorem 4.4]). Furthermore in Caffarelli's original 1977 Acta paper, he shows "almost convexity" conditions which guarantee the existence of a half ball contained in the noncontact set (see [9, Corollary 1 and Corollary 2]). Unfortunately, the union of the half balls described by Caffarelli does not contain a pseudoball of the type described in [1, so it appears that this route will not lead to a proof.

Now of course, one thing that really is shown by the argument above is that if there is a situation with $r \neq s$ and where $\partial D_{r}\left(x_{0}\right) \cap \partial D_{s}\left(x_{0}\right)$ is nonempty, then it can only happen at singular points. In this respect, and in viewing the flow of $\partial D_{t}\left(x_{0}\right)$ as we vary $t$, this situation should be compared to the results of King, Lacey, and Vázquez for the Hele-Shaw problem (see [13]). They show that if there is a corner built into the initial data, and if the angle formed satisfies certain inequalities, then that corner will remain motionless for a while at the beginning of the evolution. It is also worth observing that 
Serfaty and Serra have shown a normal velocity formula for the free boundary in the obstacle problem at regular points when varying the data, but their result (a normal velocity formula) obviously cannot be applied at a point of the free boundary that does not have a normal vector (see [17]).

Another attack which could lead to a full proof via the Hopf Lemma would be to expand the work of Figalli and Serra ([11]) to include more than the Laplacian, so that the better regularity allowed us to touch the singular set with an interior ball. Although even the Figalli/Serra results allow for some lower-dimensional "anomalous" points that would need to be handled in order to get a touching ball, so generalization and improvement would be needed for that route, and it is quite likely impossible. In any case, an examination of the methods employed in their work reveals arguments that seem to be particular to the Laplacian, and so perturbation arguments seem like a better attack as opposed to trying to do their work from scratch in a more general setting. Even though we have not successfully expanded that work, that perturbation approach is related to our third application of the main idea in this work. The difficulty there is related to the instability of singular free boundaries. Certainly it is a trivial matter to make a singular free boundary that disappears under an appropriate perturbation. For example, $u(x)=x^{2}$ satisfies $\Delta u=\chi_{\{u>0\}} f$, with $f(x) \equiv 2$, but if you raise the boundary data and/or reduce $f$ anywhere and solve the new problem, then the free boundary will disappear. That observation led us to the question of whether or not we could find a way to make specific perturbations which always led to singular points. In the third application, although we do not get results which are precise enough to allow us to generalize [11, we do successfully find a way to approximate singular free boundaries with other singular free boundaries of solutions to obstacle problems with operators with constant coefficients and which have similar boundary data, and this approximation may be of independent interest.

In all three applications what has worked is the following idea: We use the derivative of the solution to the obstacle problem as a barrier, and using that function we can come to a contradiction where a related function that we can show is nonnegative must also become negative due to standard regularity and nondegeneracy estimates that we have for the obstacle problem if the two distinct free boundaries share a common boundary point. We present three applications of this idea in this paper, and the first two are simple enough to state here: 
Theorem 1.3 (Compact Containment of Mean Value Sets, Part I). Under the assumptions of Theorem 1.1 along with the assumption that the $a^{i j}$ belong to $C^{1,1}$ the family $\left\{D_{r}\left(x_{0}\right)\right\}$ is always strictly increasing in the sense that $r<s$ implies $\overline{D_{r}\left(x_{0}\right)} \subset D_{s}\left(x_{0}\right)$.

Now within [5] part of the main result states the following:

Theorem 1.4 (Mean Value Theorem on Riemannian manifolds). Given a point $x_{0}$ in a complete Riemannian manifold $\mathcal{M}$ (possibly with boundary), there exists a maximal number $r_{0}>0$ (which is finite if $\mathcal{M}$ is compact) and a family of open sets $\left\{D_{r}\left(x_{0}\right)\right\}$ for $0<r<r_{0}$, such that

(A) $0<r<s<r_{0}$ implies, $D_{r}\left(x_{0}\right) \subset D_{s}\left(x_{0}\right)$, and

(B) $\lim _{r \downarrow 0} \max \operatorname{dist}_{x_{0}}\left(\partial D_{r}\left(x_{0}\right)\right)=0$, and

(C) if $u$ is a subsolution of the Laplace-Beltrami equation, then

$$
u\left(x_{0}\right)=\lim _{r \downarrow 0} \frac{1}{\left|D_{r}\left(x_{0}\right)\right|} \int_{D_{r}\left(x_{0}\right)} u(x) d x,
$$

and $0<r<s<r_{0}$ implies

$$
\frac{1}{\left|D_{r}\left(x_{0}\right)\right|} \int_{D_{r}\left(x_{0}\right)} u(x) d x \leq \frac{1}{\left|D_{s}\left(x_{0}\right)\right|} \int_{D_{s}\left(x_{0}\right)} u(x) d x .
$$

Finally, if $r<r_{0}$, then the set $D_{r}\left(x_{0}\right)$ is uniquely determined as the noncontact set of any one of a family of obstacle problems. In fact, as long as the set $S \subset \mathcal{M}$ is "big enough," then $D_{R}\left(x_{0}\right)$ is the noncontact set of the following obstacle problem:

$$
\begin{aligned}
\Delta_{g} u=-\chi_{\{u<G\}} R^{-n} & \text { in } S \\
u=G\left(\cdot, x_{0}\right) & \text { on } \partial S,
\end{aligned}
$$

where $G$ is the Green's function for the Laplace-Beltrami operator on $S$.

Remark 1.5 (A Few More Details). A simple criteria to determine if $S$ is "big enough" in the theorem above is to see if $\{u<G\} \subset \subset S$. Assuming that it is, then the resulting noncontact set is indeed a mean value set. A simple exercise shows that if $S$ is big enough and $S \subset S^{\prime} \subset \mathcal{M}$ then solving Equation 1.4 with $S$ replaced by $S^{\prime}$ leads to the exact same noncontact set. (The new Green's function and the new solution change by the exact same harmonic function.) In fact, it will frequently be more convenient to work with the height function, and so we define $w_{r}(x):=G\left(x_{0}, x\right)-u_{r}(x)$ which obeys either:

$$
L w_{r}=\chi_{\{w>0\}} r^{-n}-\delta_{x_{0}}
$$


or

$$
\Delta w_{r}=\chi_{\{w>0\}} r^{-n}-\delta_{x_{0}}
$$

according to which case we are currently studying. (We use $\delta_{x_{0}}$ to denote the usual delta function at $x_{0}$.)

In the current work we can show the following:

Theorem 1.6 (Compact Containment of Mean Value Sets, Part II). Under the assumptions of Theorem 1.4 the family $\left\{D_{r}\left(x_{0}\right)\right\}$ is always strictly increasing in the sense that

$$
\left(\mathrm{A}^{\prime}\right) 0<r<s<r_{0} \text { implies } \overline{D_{r}\left(x_{0}\right)} \subset D_{r}\left(x_{0}\right) \text {. }
$$

In both cases, we can say that given a point $y_{0}$ contained in a mean value set around $x_{0}$, there is a unique $r$ such that $y_{0} \in \partial D_{r}\left(x_{0}\right)$.

Acknowledgements. The authors thank Michael Hill, Rustam Sadykov, and especially Luis Silvestre for helpful discussions and valuable input.

\section{Notation, Conventions, and a Preliminary Lemma}

We will use the following basic notation and assumptions throughout the paper:

$\begin{array}{ll}\mathcal{M} & \text { a smooth connected Riemannian n-manifold } \\ g & \text { the metric for our ambient manifold } \mathcal{M} \\ \chi_{D} & \text { the characteristic function of the set } D \\ \bar{D} & \text { the closure of the set } D \\ \operatorname{int}(D) & \text { the interior of the set } D \\ \partial D & \text { the boundary of the set } D \\ \Omega(w) & \{x: w(x)>0\} \\ \Lambda(w) & \{x: w(x)=0\} \\ \mathrm{FB}(w) & \partial \Omega(w) \cap \partial \Lambda(w) \\ \operatorname{Sing}(u) & \{x \in \mathrm{FB}(u) \mid x \text { is a singular point }\} \\ \operatorname{Reg}(u) & \{x \in \mathrm{FB}(u) \mid x \text { is a regular point }\} \\ B_{r}(p) & \{x \in \mathcal{M}: \text { dist } p(x)<r\} \\ \eta_{\delta}(S) & \text { the } \delta \text {-neighborhood of the set } S \\ D_{r}(p) & \text { the Mean Value set for the point } p \text { with "radius" } r \\ \Delta_{g} & \text { the Laplace-Beltrami operator on } \mathcal{M} .\end{array}$

In particular since $D_{r}\left(x_{0}\right)=\Omega\left(w_{r}\right)$ and since we will be using $D_{e} w_{r}$ to denote the derivative of $w_{r}$ in the direction $e$, it will often be less confusing to refer to $\Omega\left(w_{r}\right)$ instead of $D_{r}\left(x_{0}\right)$. 
Throughout the paper we assume that $a^{i j}(x)$ are bounded, symmetric, and uniformly elliptic, and we define the divergence form elliptic operator

$$
L:=D_{j} a^{i j}(x) D_{i}
$$

or, in other words, for a function $u \in W^{1,2}(\Omega)$ and $f \in L^{2}(\Omega)$ we say " $L u=f$ in $\Omega$ " if for any $\phi \in W_{0}^{1,2}(\Omega)$ we have:

$$
-\int_{\Omega} a^{i j}(x) D_{i} u D_{j} \phi=\int_{\Omega} f \phi .
$$

(Notice that with our sign conventions we can have $L=\Delta$ but not $L=-\Delta$.) With our operator $L$ we let $G(x, y)$ denote the Green's function for all of $\mathbb{R}^{n}$ and observe that the existence of $G$ at least on bounded sets and in $\mathbb{R}^{n}$ when $n>3$ is guaranteed by the work of Littman, Stampacchia, and Weinberger. (See [15].) For definitions of Sobolev spaces and for standard theorems about uniformly elliptic divergence form operators on $\mathbb{R}^{n}$ we refer the reader to the excellent text by Gilbarg and Trudinger and for the situation on a Riemannian manifold we mainly use volume one of the sequence of PDE texts by Michael Taylor. (See [12] and [18].)

Finally, there is a simple lemma in [6] that we will use repeatedly, so we record it here for the reader's convenience:

Lemma 2.1 (Theorem 2.7c of [6]). Suppose that for $i=1,2$, the functions $w_{i} \geq 0$ solve the obstacle problem:

$$
\begin{aligned}
& \Delta u=\chi_{\{w>0\}} g \quad \text { in } B_{1} \\
& u=\psi_{i} \quad \text { on } \partial B_{1}
\end{aligned}
$$

where $0<\mu_{1} \leq g \leq \mu_{2}$, and $\psi_{1} \leq \psi_{2} \leq \psi_{1}+\epsilon$, then

$$
w_{1} \leq w_{2} \leq w_{1}+\epsilon
$$

and in particular

$$
\left\|w_{1}-w_{2}\right\|_{L^{\infty}\left(B_{1}\right)} \leq \epsilon
$$

\section{Proof of Compact Containment of Mean Value Sets, Part I}

We assume that $L:=\partial_{i}\left(a^{i j}(x) \partial_{j}\right)$, that $\left\|a^{i j}\right\|_{C^{1,1}}<\infty$, and as above we let $u_{r}$ denote the solution to

$$
\begin{aligned}
& L(u)=-\chi_{\{u<G\}} r^{-n} \quad \text { in } B_{M}\left(x_{0}\right) \\
& u=G\left(\cdot, x_{0}\right) \quad \text { on } \partial B_{M}\left(x_{0}\right)
\end{aligned}
$$

and let $w_{r}(x):=G\left(x, x_{0}\right)-u_{r}(x)$. 
Lemma 3.1. $L\left(D_{e} w_{r}\right)$ is a function such that,

$$
\left|L\left(D_{e} w_{r}\right)\right| \leq C(\rho)<\infty \text { in } \Omega\left(w_{r}\right) \backslash B_{\rho}\left(x_{0}\right)
$$

for any direction e and $\rho>0$ so that $\overline{B_{\rho}\left(x_{0}\right)} \subset \Omega\left(w_{r}\right)$.

Proof. Define $E:=\Omega\left(w_{r}\right) \backslash B_{\rho}\left(x_{0}\right)$ and let $\phi \in C_{0}^{\infty}(E)$.

$$
\begin{aligned}
-\int_{E} a^{i j} D_{j}\left(D_{e} w_{r}\right) D_{i} \phi d x & =\int_{E} D_{e}\left(a^{i j} D_{i} \phi\right) D_{j} w_{r} d x \\
& =\int_{E} D_{i} \phi\left(D_{e} a^{i j}\right) D_{j} w_{r} d x+\int_{E}\left(D_{e} D_{i} \phi\right) a^{i j} D_{j} w_{r} d x .
\end{aligned}
$$

On the other hand, since $D_{e} \phi \in C_{0}^{\infty}(E)$ is a permissible test function, the second integral turns out to be zero:

$$
\begin{aligned}
\int_{E}\left(D_{e} D_{i} \phi\right) a^{i j} D_{j} w_{r} d x & =\int_{E} D_{i}\left(D_{e} \phi\right) a^{i j} D_{j} w_{r} d x \\
& =-\int_{E} D_{e} \phi r^{-n} d x \\
& =\int_{E} \phi D_{e} r^{-n} d x \\
& =0 .
\end{aligned}
$$

Hence, we have $L\left(D_{e} w_{r}\right)=-D_{i}\left(D_{e} a^{i j} D_{j} w_{r}\right) \in L^{\infty}(E)$, with a uniform bound since we have excised a ball around the singularity.

Proof of Theorem 1.3. From [7] we know that $\Omega\left(w_{r}\right) \subset \Omega\left(w_{s}\right)$. Hence, we need only show that there does not exist a point $q \in F B\left(w_{r}\right) \cap F B\left(w_{s}\right)$. In order to show this we will consider the function $v:=w_{s}-w_{r}$ which satisfies:

(1) $v \geq 0$ in $B_{M}$

(2) $v=w_{s} \geq 0$ on $\partial \Omega\left(w_{r}\right)$

(3) $L v=s^{-n}-r^{-n}<0$ in $\Omega\left(w_{r}\right)$

(4) $v>0$ in $\Omega\left(w_{r}\right)$

Assume that there exists a point $q \in F B\left(w_{r}\right) \cap F B\left(w_{s}\right)$. Consider the function $D_{e} w_{r}$ for some unit vector $e$ to be chosen later. Lemma 3.1 ensures that in the set $\Omega\left(w_{r}\right) \backslash B_{\rho}$, for small $\rho$, there exists $\epsilon_{1}>0$ such that

$$
L\left(v-\epsilon_{1} D_{e} w_{r}\right)<0 \text { in } \Omega\left(w_{r}\right) \backslash B_{\rho} .
$$


Also, note that, for $\rho$ small enough, $v>0$ on $\partial B_{\rho}$ by [7, Lemma 6.2], nondegeneracy, and optimal regularity. Hence, there exists $\epsilon_{2}>0$ such that

$$
v-\epsilon_{2} D_{e} w_{r} \geq 0 \text { on } \partial B_{\rho} .
$$

Now, in fact, for any $\epsilon_{2}$ whatsoever, by standard regularity results for the obstacle problem (see [9], [10], or [6]) we automatically have

$$
v-\epsilon_{2} D_{e} w_{r}=v=w_{s} \geq 0 \text { on } \partial \Omega\left(w_{r}\right) .
$$

Then, by the Weak Maximum Principle

$$
v-\epsilon D_{e} w_{r} \geq 0 \text { in } \Omega\left(w_{r}\right) \backslash B_{\rho}
$$

for $\epsilon=\min \left\{\epsilon_{1}, \epsilon_{2}\right\}$. However, by optimal regularity and nondegeneracy we know that

$$
\sup _{B_{\delta}(q)} v \leq C_{1} \delta^{2} \text { and } \sup _{B_{\delta}(q)}\left|\nabla w_{r}\right| \geq C_{2} \delta
$$

for $\delta>0$ such that $\overline{B_{\delta}(q)} \subset B_{M}$. Therefore, for $\delta$ small enough, there exists a point $y \in B_{\delta}(q)$ and a unit vector $e$ so that

$$
v(y)-\epsilon D_{e} w_{r}(y) \leq C_{1} \delta^{2}-\epsilon C_{2} \delta<0
$$

which gives us a contradiction.

\section{Proof of Compact Containment of Mean Value Sets, Part II}

Now we turn to the proof of Theorem 1.6. Before starting, however, it is worth noting how the previous proof fails in this case. Perhaps the greatest problem is the inability to define a direction $e$ globally. Accordingly, the set $D_{r}\left(x_{0}\right) \backslash B_{\rho}\left(x_{0}\right)$ which could be huge (and therefore nowhere close to being contained within a chart of the manifold $\mathcal{M}$ ) cannot be used for our argument. We must work locally and so instead of working on $D_{r}\left(x_{0}\right) \backslash B_{\rho}\left(x_{0}\right)$, we work on $D_{r}\left(x_{0}\right) \cap B_{\delta}(q)$ where $q \in \partial D_{r}\left(x_{0}\right) \cap \partial D_{s}\left(x_{0}\right)$. On this new set, however, although we have no problem defining directions as long as $\delta$ is sufficiently small, we have a new problem of potentially having our test function being negative on parts of the boundary.

The setting we have for this section assumes that we have a point $q \in \partial D_{r}\left(x_{0}\right) \cap$ $\partial D_{s}\left(x_{0}\right)$, and a $\delta>0$ that is small enough so that

(1) $B_{\delta}(q)$ is completely contained within a single chart $(\mathcal{U}, \varphi)$ of $\mathcal{M}$, 
(2) we let $y$ be points within the original manifold, and $x$ denote points in $\varphi(\mathcal{U})$ so that $x=\varphi(y)$, and

(3) we assume that the $\varphi$ is giving us normal coordinates around $q$ and then the operator $\Delta_{g}$ can be expressed:

$$
\begin{aligned}
\Delta_{g} u(y) & =\frac{1}{\sqrt{|\operatorname{det} g(x)|}} \cdot \frac{\partial}{\partial x_{i}}\left(g^{i j}(x) \sqrt{|\operatorname{det} g(x)|} \frac{\partial}{\partial x_{j}} u(x)\right) \\
& =: g^{i j}(x) \frac{\partial}{\partial x_{i}} \frac{\partial}{\partial x_{j}} u(x)+b^{j}(x) \frac{\partial}{\partial x_{j}} u(x) \\
& =: L u(x),
\end{aligned}
$$

with $g^{i j}(p) \rightarrow \delta^{i j}$, and $b^{i}(p) \rightarrow 0$ as $p \rightarrow q$. (We are using $\delta^{i j}$ to denote the Kronecker delta.)

So the picture that we have on the manifold is given in Figure 1. In terms of a source for

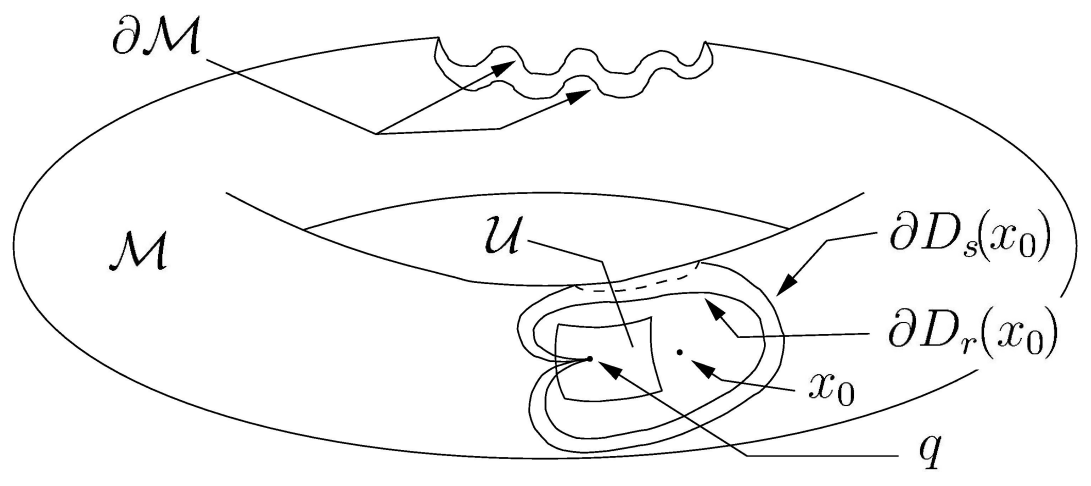

Figure 1. The Picture on the Manifold $\mathcal{M}$.

the differential geometry facts and conventions that we needed and used, we found the text [4] by Aubin to be useful for everything above.

Remark 4.1. An astute reader might complain that our mean value sets in Figure 1 lack the reflection symmetry that would be enjoyed on a piece of a perfect torus, so our picture should be considered to be a "cartoon" in this respect.

Having seen the situation on the manifold above, we observe that in this section we can do all of our work within the chart $\mathcal{U}$ and so we can view our entire problem in the local picture found in $\mathcal{V}:=\varphi(\mathcal{U}) \subset \mathbb{R}^{n}$, and this fact allows us to get away with some obvious abuses of notation. Indeed, we will use $q, D_{r}\left(x_{0}\right)$, and $D_{s}\left(x_{0}\right)$ as shorthand for $\varphi(q), \varphi\left(D_{r}\left(x_{0}\right) \cap \mathcal{U}\right)$, and $\varphi\left(D_{s}\left(x_{0}\right) \cap \mathcal{U}\right)$ respectively. Since it will be convenient to work with a perfect ball in $\mathcal{V}$, we use $B_{\epsilon}(q)$ to denote the largest ball centered at $\varphi(q)$ which 
is contained in $\varphi\left(B_{\delta}(q)\right)$. So within $\mathcal{V}$ we have a nondivergence form elliptic operator, $L$, which we can take to be defined on $C^{2}\left(\mathcal{V} \cap D_{r}\left(x_{0}\right)\right)$ and which converges to the Laplacian in the sense described above as we zoom in on $q$. Lastly, we will obviously view all of our solutions to obstacle problems (so $u_{r}$ and $w_{r}$ for example) as being functions defined on $\mathcal{V}$. All of these conventions lead to the local picture shown in Figure 2 .

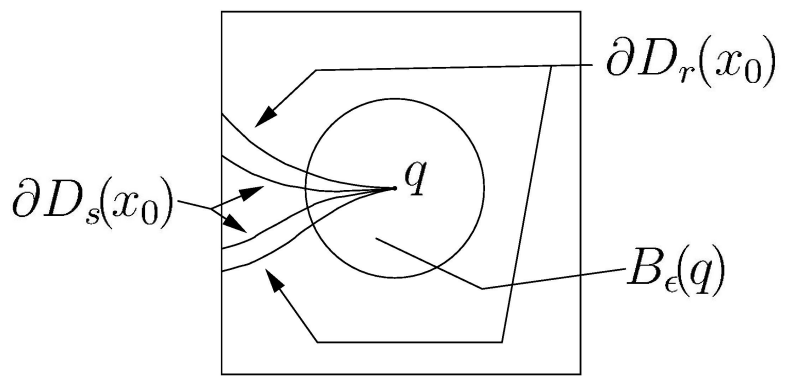

Figure 2. The Local Picture in $\mathcal{V}$.

Before jumping into the main proof, we observe the following two lemmas:

Lemma 4.2 (Barrier Function Estimates). By shrinking $\epsilon$ if necessary, we have

$$
2 n-1 \leq L\left(|x-y|^{2}\right) \leq 2 n+1,
$$

for all $x \in B_{\epsilon}(q)$ and for any fixed $y \in B_{\epsilon}(q)$.

Proof. This estimate follows immediately by using Equation (4.1) along with the fact that $g^{i j}(x) \rightarrow \delta^{i j}$ and $b^{i}(x) \rightarrow 0$ as $x \rightarrow q$.

Lemma 4.3 (Boundedness Estimate). For $x \in D_{r}\left(x_{0}\right) \cap \mathcal{V}$ and any direction e we have

$$
\left|L\left(D_{e} w_{r}\right)\right| \leq C<\infty .
$$

Proof. We observe that this estimate is very similar to the estimate in the previous section given in Lemma 3.1. On the other hand, this time the proof is easier. We know that in $D_{r}\left(x_{0}\right) \cap \mathcal{V}$ we have

$$
L w_{r}=g^{i j} D_{i j} w_{r}+b^{i} D_{i} w_{r}=r^{-n} .
$$

Differentiating this equation in the $e$ direction, we have:

$$
\begin{aligned}
0 & =D_{e}\left(g^{i j} D_{i j} w_{r}+b^{i} D_{i} w_{r}\right) \\
& =L\left(D_{e} w_{r}\right)+\left(D_{e} g^{i j}\right) D_{i j} w_{r}+\left(D_{e} b^{i}\right) D_{i} w_{r},
\end{aligned}
$$


so by using regularity known for solutions of the obstacle problem along with the regularity that we have for the coefficients in our operator $L$, we conclude that

$$
\left|L\left(D_{e} w_{r}\right)\right| \leq\left|D_{e} g^{i j}\right| \cdot\left|D_{i j} w_{r}\right|+\left|D_{e} b^{i}\right| \cdot\left|D_{i} w_{r}\right| \leq C<\infty .
$$

Proof of Theorem 1.6. We can assume by shrinking $\epsilon$ again if necessary, that $x_{0} \notin B_{\epsilon}(q)$ and $B_{\epsilon}(q)$ has no intersection with $\partial \mathcal{M}$ if $\mathcal{M}$ has boundary. (Lemma 6.2 of [7] guarantees that we can find such an $\epsilon$.) Now we consider the function

$$
h:=w_{s}-w_{r}-\mu D_{e} w_{r}
$$

where $\mu>0$ will be a very small number and $e$ will be a direction to be chosen later. We are going to arrive at a contradiction by showing that $h \geq 0$ in a ball around $q$ intersected with $D_{r}\left(x_{0}\right)$ while using the asymptotics of the functions which make up $h$ along with a good choice of the direction $e$ allow us to show that $h$ must be negative arbitrarily close to $q$ within $D_{r}\left(x_{0}\right)$.

Now for any positive $\rho<\epsilon$, we consider the set $E_{\rho}:=B_{\rho}(q) \cap D_{r}\left(x_{0}\right)=B_{\rho}(q) \cap \Omega\left(w_{r}\right)$. Within this set we have $w_{s}-w_{r} \geq 0$ and $L\left(w_{s}-w_{r}\right)=s^{-n}-r^{-n}<0$. Hence, in $E_{\rho} \backslash \eta_{\gamma}\left(\partial E_{\rho}\right)$ there exists a $\kappa$ such that $w_{s}-w_{r} \geq \kappa>0$. Having made this observation, it turns out that we will need a more precise lower bound, and by using the estimate from Lemma 4.2 we will succeed. Along these lines we first shrink $\epsilon$ (and therefore $\rho$ ) if necessary to be sure that that estimate applies, and we assume that $z \in E_{\rho} \backslash \eta_{\gamma}\left(\partial E_{\rho}\right)$ and observe that this implies that $B_{\gamma}(z) \subset E_{\rho}$. Next we define

$$
\Theta(x):=\frac{\left(s^{-n}-r^{-n}\right)\left(|x-z|^{2}-\gamma^{2}\right)}{6 n}
$$

for use as a barrier function. Indeed, observe that

(1) $\Theta=0 \leq w_{s}-w_{r}$ on $\partial B_{\gamma}(z)$, and

(2) recalling that $L\left(w_{s}-w_{r}\right)=s^{-n}-r^{-n}<0$ and using the last lemma we get:

$$
\begin{aligned}
L \Theta & =\frac{s^{-n}-r^{-n}}{6 n} \cdot L\left(|x-z|^{2}\right) \\
& \geq\left(s^{-n}-r^{-n}\right) \cdot\left(\frac{2 n+1}{6 n}\right) \\
& \geq L\left(w_{s}-w_{r}\right) \quad \text { in } \quad B_{\gamma}(z) .
\end{aligned}
$$


Thus, by using the weak maximum principle we have

$$
w_{s}(z)-w_{r}(z) \geq \frac{\gamma^{2}\left(r^{-n}-s^{-n}\right)}{6 n} \text { for all } z \text { within } E_{\rho} \backslash \eta_{\gamma}\left(\partial E_{\rho}\right) .
$$

We can now observe the following properties of $h$ :

(1) By assuming that $\mu$ is sufficiently small, we have

$$
L h=s^{-n}-r^{-n}-\mu L\left(D_{e} w_{r}\right) \leq-\alpha<0 \text { in } E_{\epsilon} .
$$

(2) $h=w_{s} \geq 0$ on $\partial \Omega\left(w_{r}\right)$.

(3) Within $E_{\rho} \backslash \eta_{\gamma}\left(\partial \Omega\left(w_{r}\right)\right)$, by assuming that $\mu$ is sufficiently small, we have

$$
h \geq \frac{\gamma^{2}\left(r^{-n}-s^{-n}\right)}{6 n}-\mu D_{e} w_{r} \geq \frac{\gamma^{2}\left(r^{-n}-s^{-n}\right)}{10 n} .
$$

(4) Within $E_{\rho} \cap \eta_{\gamma}\left(\partial \Omega\left(w_{r}\right)\right)$, by using the optimal gradient bounds for $w_{r}$, we have

$$
h \geq-\mu \gamma C .
$$

The picture can be seen in Figure 3.

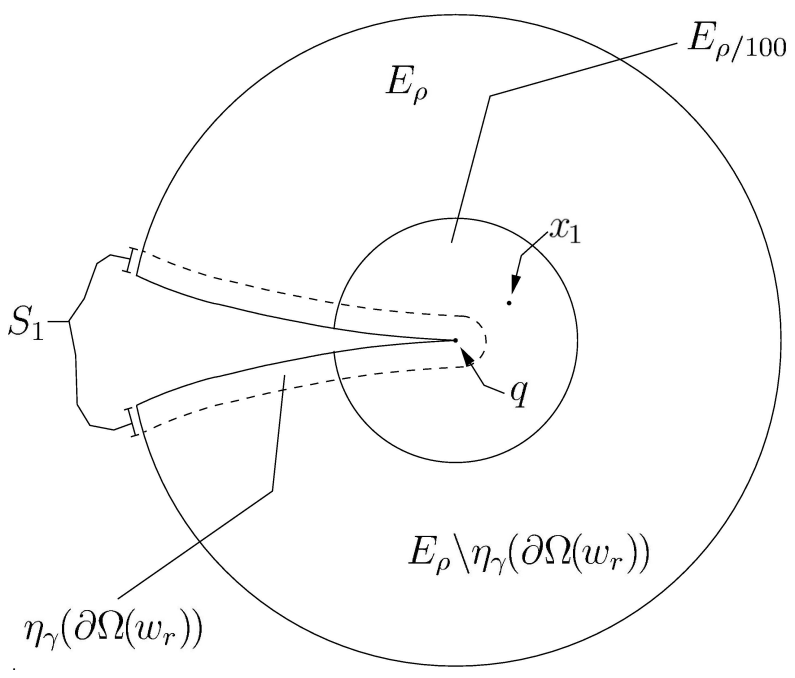

Figure 3. The Picture in $B_{\rho}(q)$

We are now in position to use the ideas within [10, Lemma 11] in order to show that $h$ must be nonnegative everywhere in a small enough ball around $q$. On the other hand, for the sake of keeping this article more self-contained, and because of slight changes that need to be made (largely because we have an operator which is close to the Laplacian, and not exactly the Laplacian) we will present the argument here. In any case we claim that $h \geq 0$ within $E_{\rho / 100}=B_{\rho / 100}(q) \cap \Omega\left(w_{r}\right)$ provided $\mu$ is sufficiently small. 
To begin the proof of our claim, we assume that there exists an $x_{1} \in E_{\rho / 100}$ with $h\left(x_{1}\right)<0$. We now define

$$
v(x):=h(x)+\delta\left(\frac{r^{-n}}{4 n}\left|x-x_{1}\right|^{2}-w_{r}\right),
$$

and we observe that $v\left(x_{1}\right)<0$. We also know that

$$
L v \leq-\alpha+\delta\left(\frac{r^{-n}}{4 n}(2 n+1)-r^{-n}\right) \leq-\alpha<0
$$

in all of $E_{\epsilon}$ by using Lemma 4.2 and Equation 4.5 . So, by applying the weak maximum principle, we can be sure that $v$ must attain a negative minimum on $\partial E_{\rho}$. On the other hand, all along $\partial \Omega\left(w_{r}\right)$, by using the definition of $h(x)$ we have $v(x)=w_{s}(x)+C \delta \mid x-$ $\left.x_{1}\right|^{2}>0$. So, we know that $v(x)$ attains its negative mimimum on $\partial B_{\rho}(q) \cap \partial E_{\rho}$. For this remaining piece of the boundary, it is convenient to split it into $S_{1}:=\eta_{\gamma}\left(\partial \Omega\left(w_{r}\right)\right) \cap \partial B_{\rho}(q)$ and $S_{2}:=\partial B_{\rho}(q) \backslash \eta_{\gamma}\left(\partial \Omega\left(w_{r}\right)\right)$, and then by employing Equations (4.7) and (4.6) on those sets respectively, we get:

$$
v \geq-C_{1} \mu \gamma+C_{2} \delta r^{-n} \rho^{2}-C_{3} \delta \gamma^{2} \quad \text { on } S_{1}
$$

and

$$
v \geq C_{4}\left(r^{-n}-s^{-n}\right) \gamma^{2}+\delta\left(C_{5} r^{-n} \rho^{2}-w_{r}\right) \quad \text { on } S_{2} .
$$

On both sets we wish to choose constants so that $v$ is forced to be nonnegative. For $S_{1}$ we choose $\gamma \ll \rho$ to force $C_{2} r^{-n} \rho^{2}>C_{3} \gamma^{2}$ and then choose $\mu$ as small as we need to give us the desired inequality. For $S_{2}$ we choose $\delta \ll \gamma^{2}$ and then shrink $\mu$ again if needed to fix the inequality on $S_{1}$. So, at this point we have a contradiction to any negativity of $v$ within $E_{\rho / 100}$.

Now, just as in the end of the proof of Theorem 1.3, it follows from standard regularity and nondegeneracy estimates for the obstacle problem, that $w_{s}$ and $w_{r}$ are bounded by a constant times $|x-q|^{2}$ within $E_{\rho / 100}$, while $D_{e} w_{r}(x)$ must grow linearly for some choice of $e$ within the same set. Now by replacing $e$ with $-e$ if necessary, we get $h<0$ somewhere within $E_{\rho / 100}$ and we have the desired contradiction.

Remark 4.4 (Existence of singular points in mean value sets). Currently, it is unknown whether or not mean value sets of the type described in the previous section ever possess singular points. So, there is an outside chance that they do not exist. Having made this observation, it is a rather simple matter to show the existence of mean value sets 
for the Laplace-Beltrami operator on manifolds which have singular points. Indeed, at the moment the topology of one of these sets changes, you will necessarily have singular points. (We can also say that by using the results within [3] the free boundary won't "jump" from a configuration with one topology where the set is smooth to a different topology with smooth boundary; there will always be a moment with a "collision.") For a concrete example, consider harmonic functions on a typical cylinder. Obviously for any such function, one can "unroll" the cylinder and get a periodic harmonic function on $\mathbb{R}^{2}$. Kuran proved that any connected mean value set for the point $x_{0} \in \mathbb{R}^{n}$ which has positive measure and which contains $x_{0}$ must be (up to a set of measure zero) a ball centered at $x_{0}([14])$. So, the $D_{r}\left(x_{0}\right)$ which fit within one period should be disks centered at $x_{0}$. By increasing the radius of the disk until the diameter is the length of a period, we get a mean value set which when viewed on the original cylinder, will have a "double cusp." Thus, we can be certain that the proof that we gave of the theorem in this section doesn't apply only to the empty set.

\section{Singular Point Approximation}

As before in Section 3 we consider an operator $L:=\partial_{i}\left(a^{i j}(x) \partial_{j}\right)$, but now, although we are still working with the obstacle problem, we are no longer working with mean-value sets, and currently we will only assume a bound on $\left\|a^{i j}\right\|_{C^{0}\left(\overline{B_{1}}\right)}$. Because our coefficients are always continuous, we can assume without loss of generality that $a^{i j}(0)=\delta^{i j}$ by changing coordinates. In this setting, we let $w \in W^{1,2}\left(B_{1}\right)$ satisfy:

$$
\left\{\begin{array}{l}
L u=\chi_{\{u>0\}} \quad \text { in } B_{1} \\
u \geq 0 \\
0 \in \operatorname{Sing}(u) .
\end{array}\right.
$$

Next, for any $r<1$, and any $t \in \mathbb{R}$, we let $u_{r ; t} \in W^{1,2}\left(B_{r}\right)$ to be the solution to

$$
\begin{cases}\Delta u=\chi_{\{u>0\}} & \text { in } B_{r} \\ u=(w+t)^{+} & \text {on } \partial B_{r} \\ u \geq 0 & \end{cases}
$$

with the goal in this section of getting $u_{r, t}$ to approximate $w$ and to also have a singular free boundary point at 0 . One reason why we had this goal, was because we had hoped to generalize the regularity results of Figalli and Serra ([11]) to obstacle problems with more general elliptic operators than simply the Laplacian. Toward this aim, we will work with quadratic rescalings of $w$ and $u_{r ; t}$, and with $T:=t / r^{2}$, we make the following list 
of definitions:

$$
\begin{aligned}
w_{r}(x) & :=\frac{w(r x)}{r^{2}} \\
v_{r ; T}(x) & :=\frac{u_{r ; t}(r x)}{r^{2}} \\
a_{r}^{i j}(x) & :=a^{i j}(r x) \\
L_{r} & :=D_{i}\left(a_{r}^{i j}(x) D_{j}\right) .
\end{aligned}
$$

Then we observe that $w_{r}$ satisfies

$$
\left\{\begin{array}{l}
L_{r} u=\chi_{\{u>0\}} \quad \text { in } B_{1} \\
u \geq 0 \\
0 \in \operatorname{Sing}(u),
\end{array}\right.
$$

and $v_{r ; T}$ is the solution to

$$
\begin{cases}\Delta u=\chi_{\{u>0\}} & \text { in } B_{1} \\ u=\left(w_{r}+T\right)^{+} & \text {on } \partial B_{1} \\ u \geq 0 . & \end{cases}
$$

Furthermore, we observe that by decreasing $r$ we can make

$$
\left\|a_{r}^{i j}(\cdot)-\delta^{i j}\right\|_{C^{0}\left(\overline{B_{1}}\right)}
$$

as small as we like.

Lemma 5.1 (Getting 0 into FB). Given $0<r \leq 1$, and defining $w_{r}$ and $v_{r ; T}$ as above, there exists an $S=S(r)$ so that $0 \in F B\left(v_{r ; S}\right)$.

Proof. We define the set $I:=\left\{T \in \mathbb{R} \mid 0 \in \operatorname{int}\left(\Lambda\left(v_{r ; T}\right)\right)\right\}$, and observe that $I$ is a bounded nonempty set. Indeed, if $T$ is sufficiently negative, then $v_{r ; T} \equiv 0$, and if $T$ is more than $1 / 2 n$, then $v_{r ; T}(x)>|x|^{2} / 2 n$. Indeed, the following inclusions follow from those observations:

$$
\left(-\infty,-\max _{B_{1}} w_{r}\right) \subset I \subset(-\infty, 1 / 2 n)
$$

So, we let $S:=\sup I$, and we claim that $0 \in F B\left(v_{r ; S}\right)$.

Suppose not. Then either $0 \in \Omega\left(v_{r ; S}\right)$ or $0 \in \operatorname{int}\left(\Lambda\left(v_{r ; S}\right)\right)$. In the first case we have a closed ball $\overline{B_{\epsilon}(0)} \subset \Omega\left(v_{r ; S}\right)$, and so in this case we let

$$
\gamma:=\min \left\{v_{r ; S}(x) \mid x \in \overline{B_{\epsilon}(0)}\right\} .
$$

Now we define $\tilde{S}:=S-\gamma / 2$ and by using Lemma 2.1 we have

$$
\gamma \leq v_{r ; S}(0) \leq v_{r ; \tilde{S}}(0)+\frac{\gamma}{2}
$$


and this inequality implies $\sup I \leq \tilde{S}<S$ which is a contradiction.

In the other case, we have a closed ball $\overline{B_{\epsilon}(0)} \subset \Lambda\left(v_{r ; S}\right)$. Now we let $T_{j} \downarrow S$ and observe that by the definition of $S$, we have $0 \in \overline{\Omega\left(v_{r ; T_{j}}\right)}$ for all $T_{j}$. Using the standard nondegeneracy results for the obstacle problem, for every $j$, we have an $x_{j}$ in $B_{\epsilon / 2}(0)$ with

$$
v_{r ; T_{j}}\left(x_{j}\right) \geq C \epsilon^{2} \text {. }
$$

This inequality leads to,

$$
C \epsilon^{2} \leq\left\|v_{r ; S}-v_{r ; T_{j}}\right\|_{L^{\infty}\left(B_{\epsilon}(0)\right)} \leq\left\|v_{r ; S}-v_{r ; T_{j}}\right\|_{L^{\infty}\left(B_{1}(0)\right)} \leq\left\|v_{r ; S}-v_{r ; T_{j}}\right\|_{L^{\infty}\left(\partial B_{1}(0)\right)},
$$

where the last inequality is by Lemma 2.1 again. However, since

$$
\left\|v_{r ; S}-v_{r ; T_{j}}\right\|_{L^{\infty}\left(\partial B_{1}(0)\right)} \leq\left\|S-T_{j}\right\|_{L^{\infty}\left(\partial B_{1}(0)\right)} \rightarrow 0
$$

we have a contradiction.

Lemma 5.2 (Uniqueness of $S$ ). The $S(r)$ given in Lemma 5.1 is the only number $S$, such that $0 \in F B\left(v_{r ; S}\right)$.

Proof. Suppose not. Then there exists $S_{1}<S_{2}$ such that $0 \in F B\left(v_{r ; S_{1}}\right) \cap F B\left(v_{r ; S_{2}}\right)$. Now from Lemma 2.1 we know that $\Omega\left(v_{r ; S_{1}}\right) \subset \Omega\left(v_{r ; S_{2}}\right)$, and we consider the function

$$
V:=v_{r ; S_{2}}-v_{r ; S_{1}}
$$

We observe that $V=S_{2}-S_{1}>0$ on $\partial B_{1} \cap \Omega\left(v_{r ; S_{1}}\right)$, and $V \in C^{0}\left(\bar{B}_{1}\right)$. By using the continuity of $V$, we have a $\delta \in(3 / 4,1)$ so that $V \geq \frac{1}{2}\left(S_{2}-S_{1}\right)$ on $\partial B_{\delta} \cap \Omega\left(v_{r ; S_{1}}\right)$. We now define the function

$$
h:=V-\mu D_{e} v_{r ; S_{1}}
$$

for a direction $e$ to be chosen later. We observe that $h$ is harmonic, and because $v_{r ; S_{1}} \in C^{1,1}\left(\overline{B_{\delta}}\right)$ we can choose $\mu$ to be sufficiently small so that $h>0$ on $\partial B_{\delta} \cap \Omega\left(v_{r ; S_{1}}\right)$. Now by the optimal regularity results for the obstacle problem $v_{r ; S_{1}}=D_{e} v_{r ; S_{1}}=0$ on all of $\partial \Omega\left(v_{r ; S_{1}}\right) \cap B_{1}$, so we observe that $h \geq 0$ on all of $\partial\left(B_{\delta} \cap \Omega\left(v_{r ; S_{1}}\right)\right)$. Thus, the maximum principle gives us $h \geq 0$ in all of $B_{\delta} \cap \Omega\left(v_{r ; S_{1}}\right)$. Now by proceeding exactly as in the proof of Theorem 1.3 where we use the asymptotics of the functions making up $h$ to find a spot where it is negative (and assigning an appropriate direction $e$ ) we get a contradiction. 
Lemma $5.3(S(r) \rightarrow 0$ as $r \rightarrow 0)$. For the $S(r)$ given in Lemma 5.1 we have

$$
\lim _{r \rightarrow 0} S(r)=0 \text {. }
$$

Proof. Suppose not. Then since the $S(r)$ are uniformly bounded, we can find a sequence $r_{j} \rightarrow 0$ such that $S\left(r_{j}\right) \rightarrow \tilde{S} \neq 0$. By our assumptions about $w_{r}$, and by applying [ , Lemma 3.1 and Lemma 3.2] we know that $w_{r_{j}} \rightarrow w_{0}=\frac{1}{2}\left(x^{T} M x\right)$ uniformly where $M$ is a nonnegative matrix and $\Delta w_{0}=\operatorname{Trace}(M)=1$. So, we know that $w_{0}$ satisfies

$$
\begin{cases}\Delta u=\chi_{\{u>0\}} & \text { in } B_{1} \\ u(x)=x^{T} M x & \text { on } \partial B_{1} \\ u \geq 0 & \end{cases}
$$

and additionally, $0 \in \operatorname{Sing}\left(w_{0}\right)$. On the other hand, since $w_{r_{j}} \rightarrow \frac{1}{2}\left(x^{T} M x\right)$ uniformly, we know that the boundary data of $v_{r_{j} ; S_{j}}$ converges uniformly to $\left(\frac{1}{2}\left(x^{T} M x\right)+\tilde{S}\right)^{+}$. So, we have that the limit of the $v_{r_{j} ; S_{j}}$, which we will call " $v_{0 ; \tilde{S}}$ " satisfies

$$
\begin{cases}\Delta u=\chi_{\{u>0\}} & \text { in } B_{1} \\ u(x)=\left(x^{T} M x+\tilde{S}\right)^{+} & \text {on } \partial B_{1} \\ u \geq 0 & \end{cases}
$$

and furthermore $0 \in \mathrm{FB}\left(v_{0 ; \tilde{S}}\right)$. Now, since $\tilde{S} \neq 0$ we can use the functions $w_{0}$ and $v_{0 ; \tilde{S}}$ along with Lemma 5.2 to get a contradiction.

Remark $5.4\left(v_{0 ; \tilde{S}}=w_{0}\right)$. It follows from knowing that $\tilde{S}=0$ along with Equations 5.9 and (5.10) and the uniqueness of the solutions to such problems that $v_{0 ; \tilde{S}}=w_{0}$ and in particular $0 \in \operatorname{Sing}\left(v_{0 ; \tilde{S}}\right)$. We will use this fact in the next proof.

Finally, to strengthen the statement of our final theorem, we follow [11] and for $m \in$ $\{0,1,2, \ldots, n-1\}$ we define the $m$-th stratum of the singular set to be the subset of the singular set where the dimension of the kernel of the blow up limit is $m$.

Theorem 5.5 (Preserving the Singular Point and Bounding the Stratum). Given the function $w$, there exists an $R>0$ such that $0 \in \operatorname{Sing}\left(v_{r ; S(r)}\right)$ for all $r<R$. Furthermore, by shrinking $R$ if necessary, this singular point is in the same or lower stratum as it is with $w$. (i.e. If 0 is in the $k$-th stratum of $w$, then it will always belong to the strata for $v_{r ; S(r)}$ with $m \leq k$ for $r<R$.)

Proof. Suppose there does not exist an $R>0$ such that $0 \in \operatorname{Sing}\left(v_{r ; S(r)}\right)$ for all $r<R$. Then there exists $r_{j} \downarrow 0$ such that $0 \in \operatorname{Reg}\left(v_{r_{j} ; S\left(r_{j}\right)}\right)$ for all $j$. Fix $\epsilon>0$ to be chosen later, 
and to simplify notation, we will let $v_{j}:=v_{r_{j} ; S\left(r_{j}\right)}$ for the duration of this proof. Using our assumption, for each $r_{j}$ there exists $n_{j} \in \partial B_{1}$ so that with $P_{r_{j}}:=\max \left\{\left(x \cdot n_{j}\right), 0\right\}^{2}$ we have

$$
\frac{v_{r_{j} ; S}(\rho x)}{\rho^{2}} \rightarrow P_{r_{j}}(x) \text { uniformly as } \rho \rightarrow 0 .
$$

However, there exists a subsequence of $r_{j}$, which we denote again by $r_{j}$, such that $n_{j} \rightarrow n_{0}$ and so

$$
P_{r_{j}} \rightarrow P_{0}=\max \left\{\left(x \cdot n_{0}\right), 0\right\}^{2}
$$

uniformly. Hence, given any $\epsilon>0$ there exists an $\mathcal{R}_{1}>0$ and a $\mathcal{K}>0$ such that if $r_{j}<\mathcal{R}_{1}$ and $\rho<\mathcal{K}$, then

$$
\left\|\frac{v_{j}(\rho x)}{\rho^{2}}-P_{0}(x)\right\|_{\infty} \leq \epsilon .
$$

On the other hand $v_{j} \rightarrow w_{0}=\frac{1}{2}\left(x^{T} M x\right)$ uniformly in $\overline{B_{1}}$ by Remark 5.4, so there exists an $\mathcal{R}_{2}$ such that $r_{j} \leq \mathcal{R}_{2}$ implies

$$
\left\|\frac{v_{j}(\rho x)}{\rho^{2}}-x^{T} M x\right\|_{\infty} \leq \epsilon .
$$

By applying [19, Lemma 2] we know that there exists a constant $\gamma>0$ so that

$$
\left\|x^{T} M x-P_{0}(x)\right\|_{\infty} \geq \gamma
$$

Now we fix $\epsilon<\gamma / 2$ and use the triangle inequality combined with Equations (5.11), (5.12), and (5.13) to get a contradiction.

At this point we have the first part of the theorem. To show the second part we essentially repeat the argument but replace the use of [19, Lemma 2] with the observation that a nonnegative matrix $M$ can be approximated arbitrarily well with matrices with lower dimensional kernels, but it will stay isolated from all of the matrices with higher dimensional kernels. To be more specific, the main difference from the first part of the proof is that in place of $P_{r_{j}}$ we would have a sequence $x^{T} M_{j} x$ where the kernels of the $M_{j}$ have dimension greater than the kernel of the $M$, and this leads to a contradiction.

\section{REFERENCES}

[1] R. Alvarado, D. Brigham, V. Maz'ya, M. Mitrea, and E. Ziadé. On the regularity of domains satisfying a uniform hour-glass condition and a sharp version of the Hopf-Oleinik boundary point principle. J. Math. Sci. (N.Y.), 176(3):281-360, 2011. Problems in mathematical analysis. No. 57.

[2] Niles Armstrong. Properties of mean value sets: Angle conditions, blowup solutions, and nonconvexity. Potential Anal., 2018. Preprint. 
[3] Ashok Aryal and Ivan Blank. Mean value sets of divergence form elliptic operators and their properties. Potential Anal., 2017.

[4] Thierry Aubin. Some nonlinear problems in Riemannian geometry. Springer Monographs in Mathematics. Springer-Verlag, Berlin, 1998.

[5] B. Benson, I. Blank, and J. LeCrone. Mean value theorems for riemannian manifolds via the obstacle problem. 2018. Preprint.

[6] Ivan Blank. Sharp results for the regularity and stability of the free boundary in the obstacle problem. Indiana Univ. Math. J., 50(3):1077-1112, 2001.

[7] Ivan Blank and Zheng Hao. The mean value theorem and basic properties of the obstacle problem for divergence form elliptic operators. Comm. Anal. Geom., 23(1):129-158, 2015.

[8] Ivan Blank and Zheng Hao. Reifenberg flatness of free boundaries in obstacle problems with VMO ingredients. Calc. Var. Partial Differential Equations, 53(3-4):943-959, 2015.

[9] Luis A. Caffarelli. The regularity of free boundaries in higher dimensions. Acta Math., 139(3-4):155$184,1977$.

[10] Luis A. Caffarelli. The obstacle problem. Lezioni Fermiane. [Fermi Lectures]. Accademia Nazionale dei Lincei, Rome; Scuola Normale Superiore, Pisa, 1998.

[11] Alessio Figalli and Joaquim Serra. On the fine structure of the free boundary for the classical obstacle problem. 2018. Preprint.

[12] David Gilbarg and Neil S. Trudinger. Elliptic partial differential equations of second order. Classics in Mathematics. Springer-Verlag, Berlin, 2001. Reprint of the 1998 edition.

[13] J. R. King, A. A. Lacey, and J. L. Vázquez. Persistence of corners in free boundaries in Hele-Shaw flow. European J. Appl. Math., 6(5):455-490, 1995. Complex analysis and free boundary problems (St. Petersburg, 1994).

[14] Ü. Kuran. On the mean-value property of harmonic functions. Bull. London Math. Soc., 4:311-312, 1972.

[15] W. Littman, G. Stampacchia, and H. F. Weinberger. Regular points for elliptic equations with discontinuous coefficients. Ann. Scuola Norm. Sup. Pisa (3), 17:43-77, 1963.

[16] David G. Schaeffer. Some examples of singularities in a free boundary. Ann. Scuola Norm. Sup. Pisa Cl. Sci. (4), 4(1):133-144, 1977.

[17] Sylvia Serfaty and Joaquim Serra. Quantitative stability of the free boundary in the obstacle problem. Anal. PDE, 11(7):1803-1839, 2018.

[18] Michael E. Taylor. Partial differential equations I. Basic theory, volume 115 of Applied Mathematical Sciences. Springer, New York, second edition, 2011.

[19] Georg S. Weiss. A homogeneity improvement approach to the obstacle problem. Invent. Math., 138(1):23-50, 1999.

Kansas State University, Mathematics Department, 138 Cardwell Hall, Manhattan, KS 66506

E-mail address: niles@math.ksu.edu

Kansas State University, Mathematics Department, 138 Cardwell Hall, Manhattan, KS 66506

E-mail address: blanki@math.ksu.edu 